\title{
HPV genotyping and p16/Ki-67 test significantly improve detection rate of high-grade cervical squamous intraepithelial lesion
}

\author{
Piotr Lewitowicz ${ }^{1}$, Anna Nasierowska-Guttmejer ${ }^{1}$, Wojciech Rokita ${ }^{2,3}$, Olga Adamczyk-Gruszka ${ }^{2,3}$, \\ Stanisław Gluszek ${ }^{4}$, Magdalena Chrapek ${ }^{5}$, Małgorzata Kolos ${ }^{1}$, Agnieszka Wrona-Cyranowska ${ }^{6}$, \\ Marcin Misiek ${ }^{6}$
}

\author{
${ }^{1}$ Department of Pathology, Faculty of Medicine and Health Sciences, Jan Kochanowski \\ University, Kielce, Poland \\ ${ }^{2}$ Faculty of Medicine and Health Sciences, Jan Kochanowski University, Kielce, Poland \\ ${ }^{3}$ Department of Obstetrics and Gynecology Province Hospital, Kielce, Poland \\ ${ }^{4}$ Department of Surgery and Surgical Nursing, Faculty of Medicine and Health \\ Sciences, Jan Kochanowski University, Kielce, Poland \\ ${ }^{5}$ Department of Probability Theory and Statistics, Institute of Mathematics, \\ the Faculty of Mathematics and Natural Sciences, Jan Kochanowski University, Kielce, \\ Poland \\ ${ }^{6} \mathrm{Clinic}$ of Gynecology, Holy-Cross Oncology Centre, Kielce, Poland
}

Submitted: 12 September 2018

Accepted: 9 November 2018

Arch Med Sci 2020; 16 (1): 87-93

DOI: https://doi.org/10.5114/aoms.2018.80697

Copyright $\odot 2018$ Termedia \& Banach

\section{Abstract}

Introduction: Liquid-based cytology allows to apply modern and specific analyses of hrHPV genotyping in p16/Ki-67 test. All of these together could raise accuracy ratio for high-grade squamous intraepithelial lesion above $90 \%$. The purpose of this study was to evaluate the diagnostic accuracy of LBC, hrHPV testing, and p16/Ki-67 testing in diagnosis of high-grade cervical intraepithelial lesions.

Material and methods: The study consisted of 176 women, out of which 50 presented with HSIL (CIN2) SCC (cervical intraepithelial lesion grade 2 squamous cell carcinoma). 126 women with a negative Pap test were pooled into the second group of the study. All patients were resampled for LBC, HPV genotyping, and for the p16/Ki-67 test. The research was carried out between May and December 2017, and second sampling were taken from 1 to 4 months.

Results: We reported a strong correlation between positive Pap test and hrHPV $(p<0.05)$ that met accuracy close to $90 \%$. We noted correlations between a positive $\mathrm{p} 16 / \mathrm{Ki}-67$ with a positive Pap test: $p<0.001 ; 66 \%$ sensitivity $(95 \% \mathrm{Cl}: 51.2-78.8 \%), 87.8 \%$ specificity $(95 \% \mathrm{Cl}: 75.2-95.4 \%), 76.8 \%$ accuracy (95\% Cl: 67.2-84.7\%), and OR 13.9 (95\% Cl: 4.9-39.2), especially HSIL and HPV16: $p<0.001$; sensitivity $(95 \% \mathrm{Cl}) 64.0$, specificity $(95 \% \mathrm{Cl})$ 98.4, accuracy $(95 \% \mathrm{Cl}) 88.6$, OR $(95 \% \mathrm{Cl}) 109.3$.

Conclusions: The results of our study indicate hrHPV genotyping as a good biomarker for the triage of patients with an abnormal cytological report. In our opinion, the hrHPV test reaches the highest level of sensitivity, specificity, and accuracy, and should be considered as crucial diagnostic test in cervical screening.

Key words: cervical cancer, cancer screening, Ki67/p16, liquid base cytology, human papilomavirus.

\author{
Corresponding author: \\ Prof. Wojciech Rokita \\ $\mathrm{MD}, \mathrm{PhD}$ \\ Faculty of Medicine \\ and Health Sciences \\ Jan Kochanowski \\ University \\ al. IX Wiekow Kielc $19 \mathrm{~A}$ \\ 25-317 Kielce, Poland \\ Phone: +48 4134969 01/09 \\ Fax: +48 413496916 \\ E-mail: rokita@kielce.com.pl
}




\section{Introduction}

Over 500,000 new cases of cervical cancer are reported worldwide each year, with about 27,000 fatal outcomes [1]. However, a recent meta-analysis restricted to the population of China illustrates the clinical aspect of cervical cancer treatment strategy, and although an additional innovative pelvic hyperthermia was applied, the 3-year survival rate remained unchanged $(O R=1.17)$ [2]. In order to decrease cervical cancer incidence ratio, the screening programs have been undergoing modification for the last years. National guidelines determine the type of program, terminology, monitoring, and quality control. The implementation of such a program must fulfil many conditions such as design, planning, feasibility testing, piloting and trial launching, scaling up health service delivery, running of full-scale programme, and finally sustainability [3]. In 1988, during a meeting of the National Institutes of Health in Bethesda, Maryland, USA, a new system for reporting cervical cytology was introduced and approved - the Bethesda system (TBS), which is still a fundamental algorithm for patients' management on daily basis. In 2001 and most recently in 2014, TBS was modified, and 'undetermined significance' category and liquid-based cytology (LBC) principles were introduced [4-6]. In clinical practice, the introduction of the ASC-US, ASC-H ('atypical squamous cells cannot exclude HSIL'), and LSIL categories significantly increased the number of ambiguous results of Pap smears that needed further verification, which in turn greatly increased the cost of screening but also led to improve high-grade detection ratio [7]. At that time, the American Society for Colposcopy and Cervical Pathology (ASCCP) has accepted the strategy for clinical treatment according to the TBS [8]. This was the first time when reporting terminology was correlated with HPV biology and clinical management. The recent cutting-edge solutions like LBC, computer-assisted imaging, and HPV-testing have been implemented over the past decade; however, looking from time perspective, only hrHPV testing significantly improved the HSIL detection ratio [9-12]. The LBC has significantly decreased the ratio of inadequate smears, improved the detection of low-grade squamous intraepithelial lesion (LSIL) and ASC-US, and increased the number of colposcopy and recent intraepithelial neoplasia diagnosis. Moreover, this allowed to keep a high detection rate of HSIL, irrespectively of patient age [13-15]. Recently, the molecular status of hrHPV, especially HPV type 16 or 18 testing and prophylactic HPV vaccines, have been added to cervical cancer screening program and management guidelines. Liquid-based cytology is a technique, which has taken over conventional cytology (CC) in some countries. In Europe,
Denmark and the Netherlands have implemented LBC-based screening as first [13-16]. Technically, LBC assay takes about $50 \%$ of sample volume to prepare slides, and the remaining sample, stored in the vial as a cell suspension, can be used for immunocytochemistry, molecular testing of infectious agents, DNA cytometry, and DNA ploidy analysis.

The p16/Ki-67 test provides an insight to HPV E6 and E7 oncoproteins underlying molecular disturbances. They inhibit the suppressor genes p53 and $\mathrm{Rb}$ leading to their inactivation, respectively. In effect, the connection between E7 and Rb gene promotes cell proliferation that could be visualized by cytoplasmic $\mathrm{p} 16$ overload and Ki- 67 protein accumulation in nuclear compartment. The p16/Ki67 and hrHPV-positive tests greatly correlate with high-grade cervical intraepithelial lesion, reaching accuracy ratio over $90 \%[11,17,18]$.

This way of screening could be much more efficient than CC assuming that the specificity and sensitivity ratio of LBC and hrHPV co-tests stands for over $90 \%$. Recently ended the ATHENA trial covering above 42,000 U.S. women $\geq 25$ years compared sensitivity/ specificity of Pap cytology (ThinPrep ${ }^{\odot}$ ), hybrid strategy (cytology and HPV test), and HPV as primary screening. Conclusions pointed hrHPV testing as the most well-balanced and efficient $[9,10]$.

To reach high detection rate of HSIL, in many papers, a lot of effort has been devoted to quality control and reproducibility of molecular solutions used for HPV typing. Conclusions were mostly similar: keeping full validation from the stage of sampling through storage, adequate equipment, and analysis are crucial [19-22]. However, the relatively high-cost of testing (roughly 40 USD per singular DNA-HPV or singular p16/Ki-67 test) was pointed out and a national screening provider should face new reality by employing such modern techniques.

The aim of this study was to evaluate the diagnostic accuracy and value of LBC, hrHPV testing, and dual-stained p16/Ki-67 test in the diagnosis of high-grade cervical intraepithelial lesions including cervical cancer.

\section{Material and methods}

This regional, Polish multicenter prospective study included patients from the Clinic of Gynecology and Obstetrics, Complex Voivodship Hospital and the Department of Gynecology, HollyCross Oncology Centre in Kielce who had a CC performed. All enrolled patients were divided into two groups. The including criteria for group 1 were as follows: negative CC, negative previous screening history, and negative gynecological examination including colposcopy. Group 2 consisted of women with positive CC with cytological report covering 
ASUS-US, ASC-H, LSIL, HSIL, and SCC. As inclusion criteria included high-grade cervical neoplasia (HSIL) confirmed by cervical biopsy as spectrum lesion ranging from CIN2 to SCC, the time from CC to second sampling was from 1 to 4 months and no treatment to date. Patients who did not fulfilled the criteria were excluded.

All enrolled patients were reinvited for additional sampling for LBC, hrHPV molecular test, and p16/Ki-67 test. The research was carried out between May 2017 and December 2017, and a written consent was obtained from all participants.

The LBC were performed in the Pathology Department of Jan Kochanowski, University in Kielce using the LBC SurePath ${ }^{T M}$ system. Cytological findings were categorized according to the updated 2014 TBS protocol [6]. The total pool of 176 cases were tested for the presence of DNA hrHPV by Cobas 4800 platform (Roche Diagnostic ${ }^{\odot}$ ) using typical brushing and preservation in PreservCyt ${ }^{\oplus}$ (Roche Diagnostic ${ }^{\odot}$ ). All PCR assays were performed using Cobas $^{\odot} 4800$ commercial test (LOT Y08899). The Cobas HPV test was employed to detect $14 \mathrm{hrHPV}$ genotypes. The test specifically identifies HPV16 and HPV18, while concurrently detecting other hrHPV $(31,33,35,39,45,51$, $52,56,58,59,66$, and 68) at clinically relevant infection levels. All group No 2 patients and additional patients from group No 1 with negative cytology but positive Cobas test were assayed with TriPath SurePath ${ }^{\mathrm{TM}}\left(\mathrm{BD}^{\odot}\right)$ unstained and tested immunocytochemically with the p16/Ki-67 test (CINtec PLUS, Roche Diagnostic ${ }^{\odot}$ kit C.N 605-100, p16 IgG2a, Ki-67 lgG) according to fully validated protocol on Benchmark XT (Roche Diagnostic ${ }^{\odot}$ ). All employed solutions met the criteria of CE IVD (EU directive for the 'in-vitro-diagnostic medical devices') and FDA (the Food and Drug Agency), and are used in many national screening programs worldwide. SurePath ${ }^{\odot}$ LBC and hrHPV Cobas $^{\odot}$ tests were performed in every case. One patient was excluded from the study due to an invalid PCR test.

LBC and p16/Ki-67 immunocytochemical reactions were analyzed by three independent pathologists ( $\mathrm{PL}, \mathrm{MK}, \mathrm{AN}-\mathrm{G}$ ) blinded to the clinical data. All observed data was tabulated and then statistically analyzed. All inter-observer discrepancies were collectively reanalyzed and to final calculations consensus results were used.

\section{Statistical analysis}

Categorical data was expressed as number and percentage. Chi-square $\left(\chi^{2}\right)$ test or Fisher's exact test were applied to compare proportions. Numerical variables were presented as median and interquartile range and compared by Mann-Whitney $U$ test. Interobserver variabilities were assessed by Cohen's $\kappa$ coefficient. Sensitivity, specificity, ac- curacy, and odds ratio (OR) with 95\% confidence intervals $(95 \% \mathrm{Cl})$ were also calculated. A two tailed $p$-value $<0.05$ was considered as statistically significant. All statistical analyses were performed using R (version 3.1.2; the R Foundation for Statistical Computing, Vienna, Austria) or Statistica (StatSoft, Inc., 2014, version 12).

\section{Results}

One hundred and seventy five women ranging from 21 to 67 years of age (average age: 37.5; STD, 10.4; median, 34) were included in the analysis. Group 1 consisted of 126 women, age ranging from 21 to 67 (average: 38.3 years). Group 2 included 50 patients, with age ranging from 23 to 59 (average: 35.3 years). There were no statistical differences between the groups according to age $(p=0.14)$. Applied solution resulted in $0.5 \%$ ratio of unsatisfactory slides and $8 \% \mathrm{HSIL}$ underdiagnosis by CC versus LBC. Blinded to clinical data pathologists reached $62 \%$ specificity to detect HSIL. The remaining 19 patients were coded as non-HSIL spectrum lesions. Table I presents the comparison of HPV infection and Pap test findings. Every categorized HPV type strongly associated with positive cytology confirming its strong value as biomarker for intraepithelial lesion. Inside both LSIL and unequivocal pattern group (ASC-US, ASC-H, AGUS), 84\% frequency ratio of hrHPV infection was found.

Table II presents high percentage of sensitivity, specificity, and accuracy for hrHPV. In HPV16 and generally all types of HPV infection met accuracy close to $90 \%$. Moreover, we observed high specificity of all types of HPV infection as predictors of cell abnormality as cancer prodromal sign.

To evaluate the utility of p16/Ki-67 in HSIL, we compared 64 pooled cases including 50 women with positive Pap test and 14 hrHPV-positive patients with negative Pap test; CIN excluded by biopsy (grey zone). The control group consisted of 36 triple negative (negative cytology, clinical examination, and Cobas test) cases. Full inter-observer agreement reached 90.9\% (90/99), while Cohen's $\kappa$ coefficient was 0.807 . Strong correlations were observed between a positive p16/ Ki-67 and a positive Pap test (66\% sensitivity (95\% Cl: $51.2-78.8 \%), 87.8 \%$ specificity $(95 \% \mathrm{Cl}$ : 75.2-95.4\%), 76.8\% accuracy (95\% Cl: 67.2-84.7\%), and $\mathrm{OR}=13.9(95 \% \mathrm{Cl}: 4.9-39.2 ; p<0.001)$, especially HSIL and HPV16 (sensitivity $(95 \% \mathrm{Cl})$ 64.0, specificity $(95 \% \mathrm{Cl}) 98.4$, accuracy $(95 \% \mathrm{Cl})$ 88.6, OR $(95 \% \mathrm{Cl}) 109.3 ; p<0.001)$. HPV 16 was found more frequent in the HSIL $(p=0.026)$ and collaterally positive $\mathrm{p} 16 / \mathrm{Ki}-67$ test proved of strong diagnostic predictor of HSIL ( $p=0.002$, Table III).

The positive Pap test, confirmed by biopsy as CIN2-SCC, could be the golden standard to esti- 
Table I. Comparison of HPV genotype with HSIL and non-HSIL lesions

\begin{tabular}{|c|c|c|c|c|c|c|c|}
\hline \multirow[t]{3}{*}{ Variable } & \multirow[t]{3}{*}{$\begin{array}{c}\text { All } \\
(n=175)\end{array}$} & \multicolumn{4}{|c|}{$\begin{array}{l}\text { Positive Pap test } \\
\quad(n=50)\end{array}$} & \multirow{3}{*}{$\begin{array}{l}\text { Negative } \\
\text { Pap test } \\
(n=125)\end{array}$} & \multirow[t]{3}{*}{$P$-value* } \\
\hline & & \multicolumn{3}{|c|}{ Collectively as non HSIL $(n=19)$} & \multirow{2}{*}{$\begin{array}{c}\text { HSIL } \\
(n=31)\end{array}$} & & \\
\hline & & $\begin{array}{l}\text { ASC-US } \\
(n=10)\end{array}$ & $\begin{array}{l}\text { ASC-H } \\
(n=5)\end{array}$ & $\begin{array}{l}\mathrm{LSIL} \\
(n=4)\end{array}$ & & & \\
\hline HPV16: & & & & & & & $\begin{array}{l}<0.001 \\
\chi^{2} \text { test }\end{array}$ \\
\hline Positive & $34(19.4)$ & $4(20)$ & 0 & $2(50)$ & $26(76)$ & $2(1.6)$ & \\
\hline Negative & $141(80.6)$ & & & & & $123(98.4)$ & \\
\hline HPV18: & & & & & & & $\begin{array}{c}0.022 \\
\text { Fisher's } \\
\text { exact test }\end{array}$ \\
\hline Positive & $3(1.71)$ & $1(10)$ & 0 & 0 & $2(6)$ & $0(0.00)$ & \\
\hline \multirow[t]{2}{*}{ Negative } & $172(98.3)$ & & & & & $125(100)$ & \\
\hline & & & & & & & $\begin{array}{l}0.003 \\
\chi^{2} \text { test }\end{array}$ \\
\hline Positive & $28(16.0)$ & $6(60)$ & $3(60)$ & $2(50)$ & $1(3)$ & $13(10.4)$ & \\
\hline Negative & $147(84.0)$ & & & & & $112(89.6)$ & \\
\hline HPV findings: & & & & & & & $\begin{array}{c}<0.001 \\
\text { Fisher's } \\
\text { exact test }\end{array}$ \\
\hline HPV DNA not detectable & $119(68.0)$ & $1(10)$ & $2(20)$ & & $2(6)$ & $111(88)$ & \\
\hline Single HPV type & $47(26.9)$ & $9(90)$ & $3(40)$ & & $22(70)$ & $13(10.4)$ & \\
\hline HPV coinfection & $9(5.14)$ & 0 & 0 & $1(25)$ & $7(22)$ & $1(0.80)$ & \\
\hline
\end{tabular}

${ }^{*}$-value corresponds to comparison HSIL and non-HSIL versus negative Pap group; Fisher's exact test was used if any expected counts were less than 5.

Table II. Estimation of Cobas HPV categories diagnostic value

\begin{tabular}{|lcccc|}
\hline Parameter & HPV16 & HPV18 & HPV other & $\begin{array}{c}\text { HPV-positive including } \\
\text { coinfection* }^{*}\end{array}$ \\
\hline $\begin{array}{l}\text { Sensitivity } \\
(95 \% \mathrm{Cl})\end{array}$ & $64.0(49.2-77.1)$ & $6(1.3-16.5)$ & $30.0(17.9-44.6)$ & $84.0(70.9-92.8)$ \\
\hline $\begin{array}{l}\text { Specificity } \\
(95 \% \mathrm{Cl})\end{array}$ & $98.4(94.3-99.8)$ & $100(97.1-100.0)$ & $89.6(82.9-94.3)$ & $88.8(81.9-93.7)$ \\
\hline $\begin{array}{l}\text { Accuracy } \\
(95 \% \mathrm{Cl})\end{array}$ & $88.6(82.9-92.9)$ & $73.1(65.9-79.6)$ & $72.6(65.3-79.0)$ & $87.4(81.6-92.0)$ \\
\hline OR $(95 \% \mathrm{Cl})$ & $109.3(24.1-495.8)$ & - & $3.7(1.6-8.5)$ & $41.6(16.3-106.4)$ \\
\hline
\end{tabular}

- not applicable due to specificity $=100 \%$; ${ }^{*}$ cases with HPV coinfection also many types infection in category 'HPV other'.

mate HSIL detection. Our data concerning p16/Ki67 test efficiency was as follows: $66 \%$ sensitivity (95\% Cl: $51.2-78.8 \%), 87.8 \%$ specificity $(95 \% \mathrm{Cl}$ : $75.2-95.4 \%), \quad 76.8 \%$ accuracy $(95 \% \mathrm{Cl}: 67.2-$ $84.7 \%)$, and $\mathrm{OR}=13.9$ (95\% Cl: 4.9-39.2). The highest percentage of positive p16/Ki-67 tests was observed in patients with positive cytology and positive hrHPV $(p<0.001)$ (Figure 1).

\section{Discussion}

The past decade has been fruitful in attempts to improve cervical cancer screening productivity.
It seems that CC has been irretrievably replaced by much more efficient LBC and additional molecular and immunological tests.

In our SurePath study, we met $0.5 \%$ unsatisfactory slides and $8 \%$ HSIL under-diagnosis by CC versus LBC. The Dutch study by Rozemeijer et al. published a large-cohort studies demonstrating the advantage of LBC over CC. The authors compared the sensitivity of CC, SurePath, and ThinPrep methods. They observed a $14 \%$ improvement in the detection of CIN I and CIN II using SurePath vs. CC. ThinPrep did not improve the CIN detection rate. In more recent study, HR at 0.81 was associ- 
Table III. Comparison of HSIL and non-HSIL detection rate according to HPV genotypes and p16/Ki-67 test

\begin{tabular}{|c|c|c|c|c|c|c|c|}
\hline \multirow[t]{3}{*}{ Variable } & \multicolumn{2}{|c|}{ Control group } & \multicolumn{4}{|c|}{ Targeted group } & \multirow[t]{3}{*}{$P$-value* } \\
\hline & \multirow{2}{*}{$\begin{array}{l}\text { Absolute } \\
\text { control } \\
\text { group } \\
(n=36)\end{array}$} & \multirow{2}{*}{$\begin{array}{l}\text { Grey zone } \\
(n=14)\end{array}$} & \multirow{2}{*}{$\begin{array}{c}\text { HSIL } \\
(n=31)\end{array}$} & \multicolumn{3}{|c|}{ Collectively as non-HSIL $(n=19)$} & \\
\hline & & & & $\begin{array}{l}\text { ASC-US } \\
(n=10)\end{array}$ & $\begin{array}{l}\text { ASC-H } \\
(n=5)\end{array}$ & $\begin{array}{c}\mathrm{LSIL} \\
(n=4)\end{array}$ & \\
\hline $\begin{array}{l}\text { Age median } \\
\text { Q1; Q3 }\end{array}$ & \multicolumn{2}{|c|}{$34.0(29.0 ; 39.5)$} & $\begin{array}{c}33.0 \\
(27.5 ; 37.0)\end{array}$ & \multicolumn{3}{|c|}{$35.0(31.5 ; 42.0)$} & $\begin{array}{c}0.133 \\
\text { Mann- } \\
\text { Whitney } \\
U \text { test }\end{array}$ \\
\hline HPV16: & & & & & & & 0.026 \\
\hline Negative & \multicolumn{2}{|c|}{$18(36.0)$} & $7(22.6)$ & & & & \\
\hline Positive & \multicolumn{2}{|c|}{$32(64.0)$} & $24(77.4)$ & $2(10)$ & 0 & $2(50)$ & \\
\hline \multicolumn{3}{|l|}{ HPV18: } & & & & & 1.000 \\
\hline Negative & \multicolumn{2}{|c|}{$47(94.0)$} & $29(93.5)$ & & & & \\
\hline Positive & \multicolumn{2}{|c|}{$3(6.00)$} & $2(6.45)$ & $1(10)$ & 0 & 0 & \\
\hline \multicolumn{3}{|l|}{ HPV other: } & & & & & 0.611 \\
\hline Negative & \multicolumn{2}{|c|}{$35(70.0)$} & $23(74.2)$ & & & & \\
\hline Positive & \multicolumn{2}{|c|}{$15(30.0)$} & $8(25.8)$ & $6(60)$ & $3(60)$ & $2(50)$ & \\
\hline \multicolumn{3}{|l|}{ p16/Ki-67: } & & & & & 0.002 \\
\hline Negative & \multicolumn{2}{|c|}{$17(34.0)$} & $5(16.1)$ & 9 & 4 & 0 & \\
\hline Positive & \multicolumn{2}{|c|}{$33(66.0)$} & 26 (83.9) & $1(36.8)$ & 1 & 4 & \\
\hline \multicolumn{3}{|l|}{ HPV and p16/Ki-67**: } & & & & & $0.003^{* *}$ \\
\hline $\begin{array}{l}\text { HPV-negative } \\
\text { p16/Ki-67-negative }\end{array}$ & \multicolumn{2}{|c|}{40} & $2(6.45)$ & $1(10)$ & $2(40)$ & 0 & \\
\hline $\begin{array}{l}\text { HPV-negative } \\
\text { p16/Ki-67-positive }\end{array}$ & \multicolumn{2}{|c|}{3} & $1(3.23)$ & 0 & 1 & 0 & \\
\hline $\begin{array}{l}\text { HPV-positive } \\
\text { p16/Ki-67-negative }\end{array}$ & \multicolumn{2}{|c|}{15} & $3(9.68)$ & $9(90)$ & 0 & 0 & \\
\hline $\begin{array}{l}\text { HPV-positive } \\
\text { p16/Ki-67-positive }\end{array}$ & \multicolumn{2}{|c|}{34} & $25(80.6)$ & 0 & $2(40)$ & $4(100)$ & \\
\hline
\end{tabular}

${ }^{\star}$ Comparative analysis of HSIL, non-HSIL with double negative group (negative Pap test with negative HPV test). ${ }^{* *}$ Comparative analysis of HSIL detection by $16 / \mathrm{Ki}$-67 test according to HPV-positive test.

ated with a $19 \%$ lower risk of cervical cancer using SurePath vs. CC. This large cohort study covered most parts of The Netherlands, and we reached a similar value of specificity at slightly above $60 \%$ $[13,14]$. Similarly, Beerman et al. reported higher detection of ASC-US+ (2.97\% vs. $1.64 \%, p<0.001)$ and a lower rate of unsatisfactory slides with SurePath compared to CC $(0.13 \%$ vs. $0.89 \%, p<$ 0.001 ) [15]. In light of our observation, high-grade lesions were masked by ASC-US or ASC-H, which proves missing many HSIL using CC only. Table II shows a $42 \%$ ratio of $\mathrm{HSIL}$ underdiagnosis by $\mathrm{LBC}$ and instead of HSIL, the LSIL, ASC-US, and ASC-H were diagnosed.

We noted a strong correlation between HPV16 and 'HPV other' with positive cytological findings. Furthermore, a positive p16/Ki-67 test strongly correlated with HPV16, particularly with HSIL $(p<00001)$. These results confirm the crucial

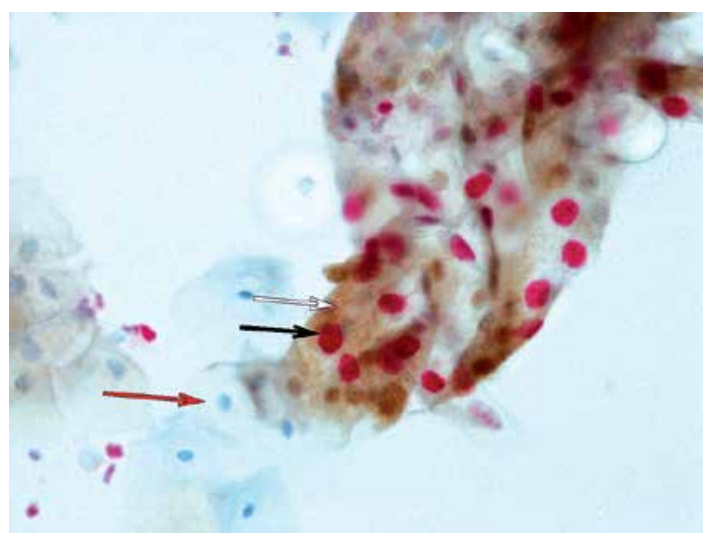

Figure 1. SurePath based on the p16/Ki-67 immunocytochemical assay: strongly positive reaction with LSIL pattern. Brown cytoplasmic chromogen corresponds to p16 overload (white arrow); nuclear compartment red chromogen is a proof of high Ki-67 protein accumulation and proliferative activity (black arrow); red arrow depicts unaffected cell (case of HPV multi-infection), magnification 40x 
role of HPV16 of cancer detecting. Our results confirmed the efficiency of the p16/Ki-67 test in triaging HPV16 -positive cases $(p=0.026)$. Similar observations were reported by Rokita et al. who found a very high diagnostic value of the p16/Ki-67 test in triaging patients with a positive Pap smear (ASC-US, ASC-H, LSIL) in cervical cancer screening [18]. In recent research from China, authors also reported a high correlation between HSIL and p16/Ki-67 in HPV16-positive women [11]. A fresh substudy nested in the ATHENA trial was also reported the utility p16/Ki-67. Wright et al. noted higher sensitivity of the $\mathrm{p} 16 / \mathrm{Ki}-67$ test compared to Pap cytology for triaging HPV-positive women $(p<0.0001)$ [9]. The same results were reported by Posatti-Resende et al. and Ordi et al. [17, 23].

Many molecular analyses for the HPV infection detection are available. The most widely studied include $\mathrm{HC2}^{\odot}$ (Qiagen), Cobas $4800^{\circ}$ (Roche), Clart $^{\odot}$ (Genomica), Aptima ${ }^{\odot}$ (Hologic), and recently launched Oncoclarity on Viper $\left(\mathrm{BD}^{\odot}\right)$. However, some discrepancies between these assays exist and there is a vide agreement about their clinical usefulness. Except for Clart, the rest of the assays have been FDA-approved for screening as reliable and high throughput solutions [19, 21, 22]. It is important to note that the preservative fluid, storage time, and additional long-term preservatives have an impact on the reliability of the results [20]. In our opinion, performing p16/Ki-67 dual staining tests is useful, even crucial, for the correct categorization of hrHPV-positive patients with unequivocal cytological report, especially LSIL, ASC-US, or ASC-H. Barron et al. published a paper devoted to triaging LSIL-H (ASC-H) using hrHPV typing. They concluded that a Pap smear categorized as LSIL-H has a significant lower hrHPV ratio than all stage above CIN2 (CIN2+) [24]. Our cohort reported 5 cases of ASC-H with a $60 \%$ frequency of 'HPV other' infection. The most recent interesting paper published by Samimi et al. reported a problem with a cytological pattern detection of HPV infection. The authors concluded the HSIL cytological pattern might be obscured in cases of multigenotype hrHPV infection and in fact, true HSIL cases could mimic unequivocal Pap patterns (ASC-US, ASC-H, LSIL) [25]. In our cohort, we found only one biopsy, which confirmed Semimi's thesis. Specifically, we discovered a case of CIN2 with HPV coinfection strongly positive with $\mathrm{p} 16 / \mathrm{Ki}-67$, but interestingly, with persistent LSIL cytological pattern. Whether this is nuclear abnormality masking or sampling error remains unclear (Figure 1). In general, we found $16 \%$ hrHPV coinfection and $2(6 \%)$ cases of HSIL hrHPV-negative on Cobas platform, proving that rarer hrHPV genotypes could be involved but they are not matched by commercial assays. According to our results, we found only
3 cases of isolated HPV18 infection with no statistical value, and the subgroup 'HPV other' did not demonstrate $\mathrm{p} 16 / \mathrm{Ki}-67$ overexpression ( $p=$ 0.502). This indirectly confirms the leading role of HPV16 what is in line with many observers. There is a question: does HPV16 E7 oncoprotein act the same way as other HPV ones?

All our calculations concerning p16/Ki-67 demonstrated a $66 \%$ sensitivity and $87.8 \%$ specificity for CIN 2+ (HSIL), which are in line with other studies $[9,11,17,18]$.

Our research was based on 50-item group and the results are consistent with other studies. The small cohort is an expected weakness of that analysis and there is a need for additional large-cohort study on Polish population. In addition, we did not reach statistical correlation with HPV18 and 'HPV other' what need further investigation, especially in light of mentioned E7 oncoprotein. Our study is considered the first paper presenting the LBCHPV-p16/Ki-67 aspect in Poland. Undoubtedly, the strong side of our study was methodology used and technical aspect in compliance with CE IVD and FDA principles.

In conclusion, the results of our study indicate hrHPV genotyping and dual-stained p16/Ki-67 testing as good biomarkers for the triage of patients with an abnormal cytological report. We noted a high efficiency of SurePath ${ }^{\odot}$ and its reliability for molecular and immunological applications. In our opinion, the hrHPV test reaches the highest level of sensitivity, specificity, and accuracy, and should be considered as a crucial diagnostic test in cervical screening. Assuming the advantage of LBC over CC and consequent higher diagnostic ratio, this method of screening should rapidly replace the CC testing. Only high-quality solutions provide globally comparable results and should be incorporated in a national screening program.

\section{Acknowledgments}

Roche Diagnostic Poland was the sponsor of the CINtec PLUS kit used for one hundred tests. Moreover, the authors are grateful to Mrs Ewa Maurycy and Mrs Ewelina Dobek, staff of JKU Pathology Department, for their excellent technical support.

The study was based on the own financial resources of the Jan Kochanowski University in Kielce, Poland.

\section{Conflict of interest}

The authors declare no conflict of interest.

\section{References}

1. Therasse P, Arbuck SG, Eisenhauer EA, et al. New guidelines to evaluate the response to treatment in solid 
tumors. European Organization for Research and Treatment of Cancer, National Cancer Institute of the United States, National Cancer Institute of Canada. J Natl Cancer Inst 2000; 92: 205-16.

2. Baoning $\mathrm{Q}$, Chuandao $\mathrm{S}$, Na S, et al. Efficacy and safety of radio-chemotherapy combined with thermotherapy for advanced cervical cancer in Chinese women: a metaanalysis. Arch Med Sci Civil Dis 2017; 2: e182-190.

3. Lynge E, Törnberg S, Von Karsa L, et al. Determinants of successful implementation of population-based cancer screening programmes. Eur J Cancer 2012; 48: 743-8.

4. Solomon D, Davey D, Kurman R, et al.; Forum Group Members; Bethesda 2001 Workshop. The 2001 Bethesda System: terminology for reporting results of cervical cytology. JAMA 2002; 287: 2114-9.

5. Solomon D, Nayar R. The Bethesda system for reporting cervical cytology. Definitions, criteria, and explanatory notes. Springer-Verlag, New York 2004.

6. Nayar D, Wilbur D. The Bethesda system for reporting cervical/vaginal cytologic diagnoses. Definitions, criteria, and explanatory notes. Springer-Verlag, New York 2015.

7. Barron S, Li Z, Austin RM, Zhao C. Low-grade squamous intraepithelial lesion/cannot exclude high-grade squamous intraepithelial lesion (LSIL-H) is a unique category of cytologic abnormality associated with distinctive HPV and histopathologic CIN 2+ detection rates. Am J Clin Pathol 2014; 141: 239-46.

8. Darragh TM, Colgan TJ, Thomas Cox J, et al. The Lower Anogenital Squamous Terminology Standardization project for HPV-associated lesions: background and consensus recommendations from the College of American Pathologists and the American Society for Colposcopy and Cervical Pathology. Int I Gynecol Pathol 2013; 32: 76-115. Erratum in: Int J Gynecol Pathol 2013; 32 432; Int J Gynecol Pathol 2013; 32: 241.

9. Wright TC Jr, Behrens CM, Ranger-Moore J, et al. Triaging HPV-positive women with p16/Ki-67 dual-stained cytology: results from a sub-study nested into the ATHENA trial. Gynecol Oncol 2017; 144: 51-6.

10. Wright TC, Stoler MH, Behrens CM, Sharma A, Zhang G, Wright TL. Primary cervical cancer screening with human papillomavirus: end of study results from the ATHENA study using HPV as the first-line screening test. Gynecol Oncol 2015; 136: 189-97.

11. Yu LL, Guo HQ, Lei XQ, et al. p16/Ki-67 co-expression associates high risk human papillomavirus persistence and cervical histopathology: a 3-year cohort study in China. Oncotarget 2016; 7: 64810-9.

12. Ronco G, Zappa M, Franceschi S, et al. Impact of variations in triage cytology interpretation on human papillomavirus-based cervical screening and implications for screening algorithms. Eur J Cancer 2016; 68: 148-55.

13. Rozemeijer K, Naber SK, Penning C, et al. Cervical cancer incidence after normal cytological sample in routine screening using SurePath, ThinPrep, and conventional cytology: population-based study. BMJ 2017; 356: 504.

14. Rozemeijer K, Penning C, Siebers AG, et al. Comparing SurePath, ThinPrep, and conventional cytology as primary test method: SurePath is associated with increased CIN II+ detection rates. Cancer Causes Control 2016; 27: 15-25.

15. Beerman H, Van Dorst EB, Kuenen-Boumeester V, Hogendoorn PC. Superior performance of liquid-based versus conventional cytology in a population-based cervical cancer screening program. Gynecol Oncol 2009; 112: 572-6.
16. Van Ballegooijen M, Hermens R. Cervical cancer screening in the Netherlands. Eur J Cancer 2000; 36: 2244-6.

17. Possati-Resende JC, Fregnani JH, Kerr LM, et al. The accuracy of p16/Ki-67 and HPV test in the detection of CIN2/3 in women diagnosed with ASC-US or LSIL. PLoS One 2015; 10: e0134445.

18. Rokita W, Kędzia W, Pruski D, et al. Comparison of the effectiveness of cytodiagnostics, molecular identification of HPV HR and CINtecPLUS test to identify LG SIL and HG SIL. Ginekol Pol 2012; 83: 894-8.

19. Rebolj M, Bonde J, Preisler S, Ejegod D, Rygaard C, Lynge E. Differential detection of human papillomavirus genotypes and cervical intraepithelial neoplasia by four commercial assays. J Clin Microbiol 2016; 54: 2669-75.

20. Gilbert L, Oates E, Ratnam S. Stability of cervical specimens in SurePath medium for human papillomavirus testing with the Roche cobas 4800 assay. J Clin Microbiol 2013; 51: 3412-4.

21. Preisler S, Rebolj M, Ejegod DM, Lynge E, Rygaard C, Bonde J. Cross-reactivity profiles of hybrid capture II, cobas, and APTIMA human papillomavirus assays: split-sample study. BMC Cancer 2016; 16: 510.

22. Fornari D, Rebolj M, Bjerregård B, et al. Hybrid capture 2 and cobas human papillomavirus assays perform similarly on SurePath samples from women with abnormalities. Cytopathology 2016; 27: 249-60.

23. Ordi J, Sagasta A, Munmany M, et al. Usefulness of p16/ $\mathrm{Ki}-67$ immunostaining in the triage of women referred to colposcopy. Cancer Cytopathol 2014; 122: 227-35.

24. Barron S, Li Z, Austin RM, Zhao C. Low-grade squamous intraepithelial lesion/cannot exclude high-grade squamous intraepithelial lesion (LSIL-H) is a unique category of cytologic abnormality associated with distinctive HPV and histopathologic CIN 2+ detection rates. Am J Clin Pathol 2014; 141: 239-46.

25. Samimi SA, Mody RR, Goodman S, et al. Do infection patterns of human papillomavirus affect the cytologic detection of high-grade cervical lesions on papanicolaou tests? Arch Pathol Lab Med 2018; 142: 347-52. 